An analysis of the modal field in absorbing optical waveguides and some useful approximations

This content has been downloaded from IOPscience. Please scroll down to see the full text. 1989 J. Phys. D: Appl. Phys. 22603

(http://iopscience.iop.org/0022-3727/22/5/006)

View the table of contents for this issue, or go to the journal homepage for more

Download details:

IP Address: 147.96.14.16

This content was downloaded on 03/02/2014 at 17:06

Please note that terms and conditions apply. 


\title{
An analysis of the modal field in absorbing optical waveguides and some useful approximations
}

\author{
Maria L Calvo and Vasudevan Lakshminarayanan $\dagger$ \\ School of Optometry, University of California, Berkeley, CA 94720, USA
}

Received 25 July 1988 , in final form 23 November 1988

Abstract. We present a mathematically exact formulation of the initial field striking the aperture of an absorbing optical waveguide, via an integral equation description. An analysis of the residual scattering by the waveguide is presented. Because of the presence of the non-negligible absorption coefficient, the solution for the fraction of the confined power is obtained by performing non-trivial integrations of Bessel and Hankel functions with complex arguments. In order to carry out a numerical analysis a specific computational method is applied by using an approximated polynomial expansion whose validity is also discussed.

\section{Introduction}

In studying the propagation of the confined energy along absorbing optical waveguides, one deals with some specific mathematical properties of the modal field. In particular, the introduction of an absorption coefficient (or extinction coefficient since the imaginary part of the refractive index is then different from zero), together with the standard transmission properties, implies new formulations for the initial field in terms of Bessel and Hankel functions with complex arguments (Calvo and Lakshminarayanan 1987). To derive explicit expressions for the fraction of the confined energy, non-trivial integrations of such functions are required. Even though there is considerable literature on absorbing waveguides such as optical photoreceptors (Enoch and Tobey 1981), irradiated dielectric waveguides (Mattern et al 1974) or photochromic guiding materials (Crow et al 1975) (all having in common the presence of absorbing properties in their structure) no extensive theory has been developed to deal with such cases. In particular, the derivation of an exact analytical expression of the initial field and studies of its mathematical properties have not been published.

We present in this paper an exact mathematical description of the initial field striking the aperture pupil of an absorbing cylindrical waveguide. Section 2 includes a background description and some comments on the integral representation. An approximation for the exact initial field is introduced by extending an earlier work due to Pask and Barrel (1981). In the same section, we also include the procedure for restrict-

$\dagger$ Reprint requests should be addresed to V Lakshminarayanan. ing the total scattered field to the forward direction. The uniqueness of the decomposition of the total field is discussed, illustrating the difficulties in obtaining approximate solutions by iterative procedures, since the convergence of the solution is not assured. The approximate solution provides a basis for justifying the existence of propagation modes in an actual waveguide. Section 3 includes a calculation of the fraction of the confined power within the waveguide. An application to the monomode case, wherein the waveguide supports the set of the fundamental modes, is discussed.

Section 4 includes the method of integration of the integrals for the propagated (transmitted) and absorbed energy. There are some difficulties due to the presence of complex arguments (arising from the nonnegligible absorption coefficient) in the Bessel and Hankel functions of zero order. The numerical evaluation of the explicit expressions is obtained after performing the convenient integrations, by applying polynomial expansions for both the real and imaginary parts of the Bessel functions of zero and first order with complex arguments. These integrations are necessary because the integral of the modulus squared of these functions represents the energy contributions inside and outside the waveguide core. The correctness of these approximations is discussed through the use of numerical analysis presented in $\$ 5$. Section 6 contains a summary and conclusions of the analysis.

\section{Background: an integral equation description of the electromagnetic field incident on the waveguide}

An incoming linearly polarised scalar optical wave, 


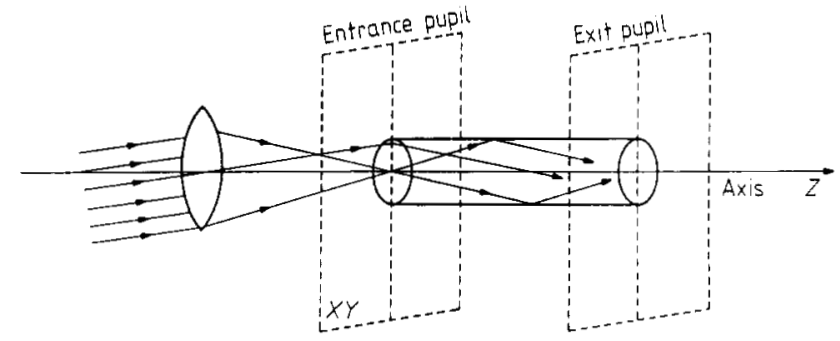

Figure 1. A schematic representation for the geometry of the problem and location of the initial field distribution at the aperture plane of the waveguide.

propagating from the remote past, interacts with the entrance pupil (plane $Z=0$ ) of an absorbing cylindrical optical waveguide. The effect of the interaction produces an additional field inside the waveguide. The mathematical form and properties of this field are related to certain restrictions involving critical confinement conditions. We shall denote by $\psi_{\text {in }}(\bar{x})$ the initial field entering into the waveguide. It propagates from plane $Z=0$ to plane $Z=Z_{0}$ (representing the finite length of the waveguide), and since the imaginary part of the complex dielectric permittivity of the waveguide is not negligible, $\hat{\varepsilon}^{i}>0$ (the real part $\hat{\varepsilon}^{\mathrm{r}}>0$ ), absorption of the penetrating field takes place together with the natural propagation (transmission) along the axial direction ( $Z$-axis). Here, and in what follows, superscripts $r$ and $i$ will denote real and imaginary parts, respectively (see figure 1 ).

Our aim is to formulate the total scattered field $\psi(\bar{x})$ following the standard three-dimensional scattering integral equation (Alvarez-Estrada and Calvo 1984):

$$
\begin{aligned}
\psi(\bar{x}) & =\psi_{\text {in }}(\bar{x})-\int_{\Omega} \mathrm{d}^{2} x^{\prime} \\
& \times \int_{0}^{Z_{0}} \mathrm{~d} Z^{\prime} K\left(\boldsymbol{x}, \boldsymbol{x}^{\prime}, Z-Z^{\prime}\right) \psi\left(\bar{x}^{\prime}\right)
\end{aligned}
$$

where $\Omega$ is the waveguide cross section. Equation (1) is satisfied by the total wavefunction of the electromagnetic optical field for any $\bar{x}=\left(x, Z\right.$ ) (also $\vec{x}^{\prime}=$ $\left.\left(\boldsymbol{x}^{\prime}, Z\right)\right) ; \boldsymbol{x}$ and $\boldsymbol{x}^{\prime}$ being two-dimensional vectors, $\boldsymbol{x}=$ $(x, y)$ and $x^{\prime}=\left(x^{\prime}, y^{\prime}\right)$.

The nucleus $K$ of the integral equation in equation (1) is defined as the product of the propagator (in the form of a standard three-dimensional Green function $G)$ and a complex function involving the optical properties of the waveguide:

$$
K\left(\boldsymbol{x}, \boldsymbol{x}^{\prime}, Z-Z^{\prime}\right)=G\left(\boldsymbol{x}-\boldsymbol{x}^{\prime}, Z-Z^{\prime}\right) k^{2}\left(\hat{\varepsilon}(\boldsymbol{x})-\varepsilon_{\mathrm{c}}\right)
$$

with

$$
\begin{array}{r}
G\left(x-x^{\prime}, Z-Z^{\prime}\right)=(1 / 2 \pi)^{3} \int \mathrm{d} \boldsymbol{l} \mathrm{d} l_{z} \\
\times \frac{\exp \left\{\mathrm{i}\left[\boldsymbol{l}\left(\boldsymbol{x}-\boldsymbol{x}^{\prime}\right)+l_{z}\left(Z-Z^{\prime}\right)\right]\right\}}{\boldsymbol{l}^{2}+\boldsymbol{l}_{z}^{2}-\left(K^{2}+\mathrm{i} \eta\right)}
\end{array}
$$

where $\bar{l}$ is a vector for integration $\left(\bar{l}=\left(l, l_{z}\right)\right), k$ is the wavenumber associated with the incoming optical field, $\hat{\varepsilon}$ is the complex dielectric permittivity of the waveguide core and $\varepsilon_{\mathrm{c}}$ is the real dielectric permittivity of the surrounding space or cladding region. $\eta$ is an infinitesimal quantity such that $\eta>0$ and $\eta \rightarrow 0^{+}$after the integration. Note that

$$
\hat{\varepsilon}(\boldsymbol{x})-\varepsilon_{\mathrm{c}}=0 \quad \text { for any } \boldsymbol{x} \text { outside } \Omega .
$$

A vector extension of the scalar equation (3), using a fully transverse tensor Green function which can be applied in the formulation of the scattering of electromagnetic fields, has been given elsewhere (Calvo and Duran 1975).

Equation (1) is equivalent to the scalar wave differential equation, together with the boundary conditions determined by $\psi_{\text {in }}(x)$.

We are interested in finding the most general form for the initial field $\psi_{\text {in }}(\bar{x})$ so that the total field $\psi(x)$ reduces to a discrete superposition of generalised propagation modes for the finite length: $0<Z<Z_{0}$. Then

$$
\psi(\bar{x})=\sum_{\alpha \hat{\beta}} c_{\alpha \hat{\beta}} \exp (\mathrm{i} \hat{\beta} Z) \varphi_{\alpha}(\boldsymbol{x}) \quad 0<Z<Z_{0} .
$$

In equation (5), $\alpha=0,1,2, \ldots ; \hat{\beta}$ extends over a finite number of complex propagation constants inside the waveguide; $c_{\alpha \dot{\beta}}$ are independent constants.

The discrete sums in equation (5) extend to all values of $\alpha, \hat{\beta}$ such that $k^{2}=\hat{\beta}_{\alpha}^{2}-\chi_{\alpha}^{2}>0 ; \hat{\beta}_{\alpha}$ is a complex function with $\hat{\beta}_{\alpha}^{\mathrm{i}}>0, \hat{\beta}_{\alpha}^{\mathrm{r}}>0$; and $\hat{\chi}_{\alpha}$ is the complex modal parameter for the $\alpha$-mode. It has positive values for both the real and imaginary parts: $\hat{\chi}_{\alpha}^{\mathrm{r}}>0, \hat{\chi}_{\alpha}^{\mathrm{i}}>0$.

In turn, $\varphi_{\alpha}$ fulfils the following homogeneous integral equation (Calvo and Lakshminarayanan 1987):

$$
\begin{aligned}
& \varphi_{\alpha}(\boldsymbol{x})=(1 / 4 \mathrm{i}) \int_{\Omega} \mathrm{d}^{2} \boldsymbol{x}^{\prime} H_{0}^{(1)}\left(\mathrm{i} \hat{\chi}_{\alpha}\left|\boldsymbol{x}-\boldsymbol{x}^{\prime}\right|\right) \\
& \times k^{2}\left(\hat{\varepsilon}\left(\boldsymbol{x}^{\prime}\right)-\varepsilon_{\mathrm{c}}\right) \varphi_{\alpha}\left(\boldsymbol{x}^{\prime}\right)
\end{aligned}
$$

and

$H_{0}^{(1)}\left(\mathrm{i} \hat{\chi}_{\alpha}\left|x-x^{\prime}\right|\right)=\left(1 / \mathrm{i} \pi^{2}\right) \int \frac{\mathrm{d}^{2} \boldsymbol{q} \exp \left[\mathrm{i} \boldsymbol{q}\left(\boldsymbol{x}-\boldsymbol{x}^{\prime}\right)\right]}{\boldsymbol{q}^{2}+\hat{\chi}_{\alpha}^{2}}$

with $H_{0}^{(1)}$ being Hankel's outgoing function of zero order and first kind. It is exponentially damped for large $|\boldsymbol{x}|\left(|\boldsymbol{x}| \gg\left|\boldsymbol{x}^{\prime}\right|\right)$ as $\boldsymbol{x}^{\prime}$ varies in $\Omega$ (Morse and Feshbach 1953).

Notice that $(4 \mathrm{i})^{-1} H_{0}^{(1)}$ is a free-space Green function for $\Delta_{x}-\hat{x}_{\dot{\alpha}}^{2}$ where $\Delta_{x}=\partial^{2} / \partial x^{2}+\partial^{2} / \partial y^{2}$.

In equation (7), $q$ is a generic wavevector in two dimensions: $q=\left(q_{x}, q_{y}\right)$, so that this equation represents the plane wave integrals of the two-dimensional Green function $H_{0}^{(1)}$ in terms of its Fourier transform, $1 /\left(q^{2}+\hat{\chi}_{\hat{\alpha}}^{2}\right)$.

We shall restrict equation (1) to the finite interval $0<Z<Z_{0}$. By performing some manipulations, taking into account equations (2) and (3) together with equations (5)-(7) and the standard three-dimensional Fourier representation for $G\left(x-x^{\prime}, Z-Z^{\prime}\right.$ ) (Morse and Feshbach 1953), we can obtain the following 
expression for $\psi_{\text {in }}(\bar{x})$ for the general case of an absorbing optical waveguide:

$$
\begin{gathered}
\psi_{\text {in }}(\bar{x})=\sum_{\alpha \hat{\beta}} c_{\alpha \hat{\beta}} \int_{\Omega} \mathrm{d}^{2} \boldsymbol{x}^{\prime} k^{2}\left(\hat{\varepsilon}(\boldsymbol{x})-\varepsilon_{\mathrm{c}}\right) \varphi_{\alpha}\left(\boldsymbol{x}^{\prime}\right) \\
\times\left(\int \frac{\mathrm{d}^{2} \boldsymbol{l}}{(2 \pi)^{2}} \exp \left[\mathrm{il}\left(\boldsymbol{x}-\boldsymbol{x}^{\prime}\right)\right] \Lambda_{\alpha \hat{\beta}}(Z, \boldsymbol{l})\right)
\end{gathered}
$$

where $l=\left(l_{x}, l_{y}\right)$, with

$$
\begin{aligned}
\Lambda_{\alpha \hat{\beta}}(Z, l)=\frac{1}{2 \sigma} \\
\times\left(\frac{\exp (\mathrm{i} \sigma Z)}{\sigma-\hat{\beta}}+\frac{\exp (\mathrm{i} \sigma Z) \exp \left[\mathrm{i}(\sigma+\hat{\beta}) Z_{0}\right]}{\sigma-\hat{\beta}}\right)
\end{aligned}
$$

and

$$
\sigma=\left(k^{2}-l^{2}\right)^{1 / 2} .
$$

Notice that the second term in equation (9) reminds one that the waveguide has finite length $Z_{0}$. In some sense, it could be associated with reflection at the exit pupil (see figure 1 plane $Z=Z_{0}$ ), in order that equation (8) be a correct solution of the free wave equation. Equations (8)-(10) yield $\psi_{\text {in }}(\boldsymbol{x})$ not only for $0<Z<Z_{0}$ but also throughout all space. It is not difficult to see that for a given $Z, \psi_{\text {in }}$ is square-integrable in the whole transverse plane: $\int_{\Omega_{x}} \mathrm{~d}^{2} \boldsymbol{x}\left|\psi_{\text {in }}(\boldsymbol{x}, \boldsymbol{Z})\right|^{2}<+\infty$.

\subsection{Approximate solution for the initial field}

Let us suppose that in equation $(9),(\sigma+\hat{\beta}) Z_{0} \gg 1$ for all $l$, so that the second term vanishes exactly or is negligible. Thus, the information which remains in the formulation of $\psi_{\text {in }}$ is that contained at the entrance pupil of the waveguide. We shall outline an approximate expression for $\psi_{\text {in }}$ similar to the decomposition given by Pask and Barrell (1981).

The main contribution to the integral over $\boldsymbol{x}^{\prime}$ on the right-hand side of equation (8) is obtained for values of $l$ such that $|\boldsymbol{l}|$ is less than or of the same order as $\hat{\chi}_{\alpha}$ (but not much larger than it). Let us now assume that $k^{2}=$ $(\omega / c)^{2}$, with $\chi_{\alpha}^{2} \ll k^{2}$, implying a weakly absorbing waveguide with $\omega$ the angular frequency and $c$ the speed of light. Then

$$
(1 / 2 \sigma) \exp (\mathrm{i} \sigma Z) \simeq\{[1 /(\hat{\beta}+\sigma)] \exp (\mathrm{i} \beta Z)\} .
$$

With this approximation, equation (8) becomes

$$
\begin{aligned}
\psi_{\mathrm{in}}(\bar{x}) & \simeq-(1 / 4 \mathrm{i}) \sum_{\alpha \hat{\beta}} c_{\alpha \dot{\beta}} \exp (\mathrm{i} \hat{\beta} Z) \\
\times & \times \int_{\Omega} \mathrm{d}^{2} \boldsymbol{x}^{\prime} k^{2}\left(\hat{\varepsilon}\left(\boldsymbol{x}^{\prime}\right)-\varepsilon_{\mathrm{c}}\right) \varphi_{\alpha}\left(\boldsymbol{x}^{\prime}\right) H_{0}^{(1)}\left(\mathrm{i} \hat{\chi}_{\alpha}\left|\boldsymbol{x}-\boldsymbol{x}^{\prime}\right|\right) .
\end{aligned}
$$

Then finally according to equation (6)

$$
\psi_{\text {in }} \simeq \sum_{\alpha \hat{\beta}} c_{\alpha \dot{\beta}} \varphi_{\alpha}(x) \exp (\mathrm{i} \hat{\beta} Z) .
$$

The initial field is then a discrete superposition of modal fields, $\varphi_{a}(x)$, associated with a finite number of bound propagation modes, affected by a phase term, $\exp (i \hat{\beta} Z)$, which represents the propagation factor in the $Z$-direction. This formulation is consistent with the decomposition relation given by Pask and Barrell (1981).

For given $Z_{0}=+\infty$ and $Z$, two incoming waves, $\psi_{\text {in }}^{(1)}$ and $\psi_{\text {in }}^{(2)}$, given by equations (8) and (9), corresponding respectively to $c_{\alpha}=0$ for $\alpha \neq \alpha(1), c_{\alpha}^{(1)} \neq$ 0 and $c_{\alpha}=0$ for $\alpha \neq \alpha(2), c_{\alpha}^{(2)} \neq 0$, with $\alpha(1) \neq \alpha(2)$, are not exactly orthogonal in $\boldsymbol{x}$ :

$$
\int \mathrm{d}^{2} \boldsymbol{x}\left[\psi_{\mathrm{in}}^{(1)}\right] *\left[\psi_{\mathrm{in}}^{(2)}\right] \neq 0
$$

However, they are orthogonal when each of them is approximated in the sense of equation (12). Notice that for given $k^{2}$, the set of all initial fields $\psi_{\text {in }}$ constitutes a finite-dimensional subspace, as the finite set of constant amplitudes $c_{\alpha \hat{\beta}}$ varies.

\subsection{Restriction to the forward scattered field (trans- mitted)}

Assume $Z>Z_{0}, Z_{0}<+\infty$ in equation (5). The second term on the right-hand side of equation (1) yields the forward-scattered field $\psi_{\mathrm{tr}}$ generated by the interaction of $\psi_{\text {in }}$ with the waveguide. After additional residue integrations, it turns out to be given by the right-hand side of equation (8) provided that $\Lambda_{\alpha \hat{\beta}}$ be now replaced by

$\Lambda_{\alpha \dot{\beta}}(l, Z)_{\mathrm{tr}}=-\frac{\exp (\mathrm{i} \sigma Z)}{2(\sigma-\hat{\beta})}\left\{1-\exp \left[\mathrm{i} Z_{0}(\hat{\beta}-\sigma)\right]\right\}$.

The explicit expression for the total wavefunction for $Z>Z_{0}$ is

$$
\psi=\psi_{\text {in }}+\psi_{\text {tr }} .
$$

$\psi_{\mathrm{tr}}$ (the transmitted field) can be obtained immediately from the above results.

\subsection{The incoming and total scattered waves and a unique decomposition}

Let $\mathscr{H}_{\text {in }}$ be the space formed by all the optical wavefunctions $\psi_{\text {in }}$, given by equations $(8)-(10)$, for any $c_{\alpha \dot{\beta}}$. Let $\psi_{0}=\psi_{0}(\boldsymbol{x})$ be an arbitrary incoming optical wavefunction: $\left(\Delta-k_{0}^{2}\right) \psi_{0}=0$ for any $\bar{x}$. The equation holds for example for $\psi_{0}=\exp (\mathrm{i} k \boldsymbol{x})$. Then, one has the unique decomposition

$$
\psi_{0}=\psi_{\text {in }}+\psi_{\text {in }}^{\prime}
$$

with $\psi_{\text {in }} \in \mathscr{H}_{\text {in }}, \psi_{\text {in }}^{\prime} \notin \mathscr{H}_{\text {in }}$, and $\psi_{\text {in }}^{\prime}$ is orthogonal to all wavefunctions inside $\mathscr{H}_{\text {in }}$, along with

$$
\left(\Delta-k_{0}^{2}\right) \psi_{\text {in }}^{\prime}=0 \quad \forall x .
$$

Consequently, the total wavefunction $\psi_{\text {tot }}$ generated by the interaction of $\psi_{0}$ with the waveguide, which is a solution of equation (1) when $\psi, \psi_{\text {in }}$ are replaced by $\psi_{\text {tot }}, \psi_{0}$, can be decomposed uniquely as

$$
\psi_{\text {tot }}=\psi+\psi^{\prime}
$$


where $\psi$ is a superposition of generalised propagation modes given by equation (5) for $0<Z<Z_{0}$ and generated by $\psi_{\text {in }}$ as described above.

On the other hand, $\psi^{\prime}$ is a solution of equation (1) with $\psi_{\text {in }}$ and $\psi$ replaced by $\psi_{\text {in }}^{\prime}$ and $\psi_{\text {in }}$ respectively. It describes only the field scattered by the waveguide but not the generalised propagation modes along the latter. For instance, a plane wave such as $\psi_{0}=\exp (\mathrm{i} \boldsymbol{k x}$ ), gives rise uniquely to a superposition of propagation modes inside the waveguide: $\psi_{\text {in }} \neq 0, \psi \neq 0$, plus a pure scattering contribution: $\psi_{\text {in }}^{\prime} \neq 0, \psi^{\prime}-\psi_{\text {in }}^{\prime} \neq 0$. This one gives rise to an infinite number of radiation or unbound modes. In our approximate treatment we consider $\psi^{\prime} \simeq$ 0 , so that the total scattered field is solely equivalent to the bound modes.

We must stress the difficulties involved in obtaining $\psi$ (equation (5)) by performing directly successive iterations of equation (1) when $\psi_{\text {in }}$ is given initially through equations (8)-(10), unless important cancellations are made. The convergence of the series of iterations would be rather difficult to control. However, the analogue of equation (1) for $\psi^{\prime}$ and $\psi_{\text {in }}^{\prime}$ enables one to determine $\psi^{\prime}$ by convergent successive iterations under the condition that $k^{2}\left(\hat{\varepsilon}-\varepsilon_{\mathrm{c}}\right)$ be small.

Hence, the uniqueness in the decomposition of the incoming and total fields implies that the total scattered field will be always expressed by equations (1)-(6). The approximate solution in equation (12) will be introduced in mathematical expressions below for a direct calculation of an explicit expression of the fraction of the confined power within the waveguide.

\section{Fraction of the confined power}

The energy confined in an optical waveguide is not uniformly distributed across any transverse plane perpendicular to the waveguide axis. In general, this energy exhibits radial dependence, and the total incident field is distributed as a linear superposition of partial contributions in the form of confined modal fields or bound modes. The radial distribution depends on the geometry of the optical waveguide, as well as on the conditions of incidence of the incoming optical field interacting with the guide (Marcuse 1972). It is well established from electromagnetic theory that the fraction of modal power confined within an optical waveguide is defined as the ratio between the fraction of power confined in the finite cross section of the waveguide and the total power confined within an area (Snyder 1969):

$$
\eta_{l m}=P_{l m}^{\mathrm{F}} / P_{l m}^{\mathrm{tot}}
$$

with

$$
P_{l m}^{\mathrm{F}}=\operatorname{Re} \int_{\Omega} \mathrm{d} \Omega\left[\psi^{m^{*}}(-\mathrm{i} \alpha / \alpha z) \psi^{\mathrm{m}}\right] .
$$

The integral in equation (19) extends over a finite domain $\Omega$ in the $x$-plane, orthogonal to the waveguide axis $Z$ and coincides with the cross section of the waveguide.
For $Z>0, P_{l m}^{\mathrm{F}}$ represents the flux of energy associated with a generalised propagation mode $(\psi=$ $\left.\exp (\mathrm{i} \hat{\beta} Z) \varphi_{\alpha}(\boldsymbol{x})\right)$ or a superposition thereof, as above, and

$$
P_{l m}^{\mathrm{tot}}=\operatorname{Re} \int_{\Omega_{x}} \mathrm{~d} \Omega\left[\psi^{m^{*}}(-\mathrm{i} \partial / \partial z) \psi^{m}\right]
$$

is an integral extending over the infinite domain $\Omega_{x}$ which coincides with the whole $x$-plane. In both equations (19) and (20), $l$ and $m$ are integers $(l, m=$ $0,1,2, \ldots)$ representing the modal order and the set of confined mode order, respectively, following standard nomenclature (Marcuse 1972, Snyder 1969).

\subsection{Monomode behaviour}

Let $\alpha=0$ in equation (12) and $m=0$ in equations (18)(20). Then, the initial field is

$$
\psi_{\text {in }}^{0} \sim c_{0} \varphi_{0}(\boldsymbol{x}) \exp \left(\mathrm{i} \hat{\beta}_{0} Z\right)
$$

to be substituted in equations (18)-(20).

As discussed by Snyder and Love (1983), in a weakly absorbing waveguide with constant complex dielectric permittivity inside the core and circular cross section, the solution or the modal field can be expressed, to a good approximation, in the same way as in the case of a circular dielectric waveguide with constant and real dielectric permittivity or a step profile for the core. Then, for an $|M|$-order mode (where $M$ is an integer), in general:

$$
\varphi_{M}=\exp (\mathrm{i} M \theta) \times \begin{cases}c_{<} J_{\mathrm{M} \mid}\left(\hat{\chi}_{\alpha}^{\prime}|x|\right) & \text { for }|\boldsymbol{x}|<R \\ \mathcal{C}_{>} H_{|\mathrm{M}|}^{(1)}\left(\mathrm{i} \hat{\chi}_{\alpha}|x|\right) & \text { for }|\boldsymbol{x}|>R\end{cases}
$$

where $R$ is the radius of the waveguide cross section, $|M|=0,1,2, \ldots$ is an integer number denoting the order for the set of the confined modes, and $\theta$ is the phase constant term. $\hat{\chi}_{\alpha}^{\prime}$ and $\hat{\chi}_{\alpha}$ are complex modal parameters such that

$$
\hat{\chi}_{\alpha}^{\prime 2}+\hat{\chi}_{\alpha}^{2}=(\omega / c)\left(\hat{\varepsilon}-\varepsilon_{c}\right)
$$

for constant angular frequency $\omega . J_{|M|}$ and $H_{|M|}^{(1)}$ are standard Bessel and outgoing Hankel functions of order $|M| . c_{<}$and $c_{>}$are constant coefficients related through the boundary conditions at $|\boldsymbol{x}|=R$ :

$$
\begin{aligned}
& c_{<} J_{M \mid}\left(\hat{\chi}_{\alpha}^{\prime} R\right)=c_{>} H_{|M|}\left(\mathrm{i} \hat{\chi}_{\alpha} R\right) \\
& c_{<}\left(\mathrm{d} J_{|M|} / \mathrm{d}|\boldsymbol{x}|\right)_{|x|=R}=c_{>}\left(\mathrm{d} H_{|M|} / \mathrm{d}|\boldsymbol{x}|\right)_{|x|=R} .
\end{aligned}
$$

$\hat{\chi}_{\alpha}^{\prime}$ is a complex number with $\hat{\chi}_{\alpha}^{\prime r}$ and $\hat{\chi}_{\alpha}^{\prime i}$ real and positive numbers.

By using equation (24), one gets the dispersion relation for the propagation modes, which, combined with equation (23) determines the modal parameters $\hat{\chi}_{\alpha}^{\prime}$ and $\hat{\chi}_{\alpha}$.

The cut-off conditions are obtained through the values of $R$ for which propagation modes with $M \neq 0$ are allowed. Then, for a given $M \neq 0$ and a fixed value of $R$, one has the necessary condition $\hat{\chi}_{\alpha}=0$. 
$\left(\hat{\chi}_{\alpha}^{\prime}=0, \hat{\chi}_{\alpha}^{\mathrm{i}}=0\right)$ for the propagation of the $|M|$ th-order mode.

A specific feature in the solution of equation (22) is that the argument of both the Bessel and Hankel functions is a complex variable. At this point, we recall that for pure dielectric optical waveguides the solutions for the modal fields are given in terms of real modal parameters. The present formulation implies that a new type of integration needs to be performed to calculate the fraction of the confined power $\eta$ defined by equation (18).

According to equations (18)-(22), the fraction of power confined within the monomode waveguide is

$$
P_{0}^{\mathrm{F}}=2 \pi\left|c_{\stackrel{(0)}{<}}\right|^{2} \exp \left(-2 \hat{\beta}_{0}^{\mathrm{i}} Z\right) \operatorname{Re}\left(\hat{\beta}_{0} I_{J}\right)
$$

with

$$
I_{J}=\int_{0}^{R} \mathrm{~d} r\left|J_{0}\left(\hat{\chi}_{0}^{\prime} r\right)\right|^{2} \quad r=|\boldsymbol{x}|
$$

and $\hat{\chi}_{0}=\hat{\chi}_{0}^{\mathrm{r}}+\mathrm{i} \hat{\chi}_{0}^{\mathrm{i}}$ is the complex parameter for the propagation of the set of confirmed modes of zero order or the fundamental modes.

The total fraction of confined power is now

$$
P_{0}^{\text {tot }}=2 \pi \exp \left(-2 \hat{\beta}_{0}^{\mathrm{i}} Z\right) \operatorname{Re}\left[\hat{\beta}_{0}\left(\left|c_{\llcorner}^{(0)}\right|^{2} I_{J}+\left|c_{>}^{(0)}\right|^{2} I_{H}\right)\right]
$$

with

$$
I_{H}=\int_{R}^{\infty} r \mathrm{~d} r\left|H_{0}^{(1)}\left(\mathrm{i} \hat{\chi}_{0} r\right)\right|^{2}
$$

and $I_{J}$ as given in equation (26). Both integrals in equations (26) and (28) must be solved to obtain an explicit expression for the fraction of the confined power within the monomode absorbing waveguide. It should be noted that the absolute magnitudes of $c_{<}^{(0)}$ and $c_{>}^{(0)}$ are not particularly necessary in evaluating $\eta$. Only the ratio $c_{<}^{(0)} / c_{>}^{(0)}$ is relevant.

\section{The integration of $I_{J}$ and $I_{H}$ : methods of cal- culation}

\subsection{General definitions}

We shall make use of the indefinite integrals of products of cylindrical functions (equation 11.3.29 of Abramowitz and Stegun (1972), Bateman Manuscript (1954)). In this case we shall interpret the general integration as follows:

$$
\begin{aligned}
& \int^{Z} C_{\mu}(k t) D_{\nu}(l t) t \mathrm{~d} t=\left(z / k^{2}-l^{2}\right) \\
& \quad \times\left[C_{\mu+1}(k z) D_{\nu}(l z)-l C_{\mu}(k z) D_{\nu+1}(l z)\right]+\Lambda
\end{aligned}
$$

where $\Lambda$ is a constant of integration.

We will also consider the following specifications in equation (29): $\mu=\nu=0, t=r, k=\hat{\chi}_{0}^{\prime}, l=\hat{\chi}_{0}^{* *}, z=R$ (and the same for $\hat{\chi}_{0}$ and $\hat{\chi}_{0}^{*}$ ). The superscript $*$ denotes the complex conjugate.
Let $C, D=J_{0}$ in equation (29) (for the $I_{J}$ case). For $z=R \rightarrow 0$

$$
I_{J}=\int_{0}^{z} C_{\mu}(k t) D_{\nu}(l t) t \mathrm{~d} t \rightarrow 0
$$

and

$$
\left(z / k^{2}-l^{2}\right)\left[C_{\mu+1}(k z) D_{\nu}(l z)-C_{\mu}(k z) D_{\nu+1}(l z)\right] \rightarrow 0
$$

as both $J$ and $J_{0}$ are finite for $z=R \rightarrow 0$. Then $\Lambda=0$.

\section{Property 1 .}

$\left|J_{0}\left(\hat{\chi}_{0}^{\prime} r\right)\right|^{2}=J_{0}\left(\hat{\chi}_{0}^{\prime} r\right) \cdot \overline{J_{0}\left(\hat{\chi}_{0}^{\prime} r\right)}=J_{0}\left(\hat{\chi}_{0}^{\prime} r\right) \cdot J_{0}\left(\hat{\chi}_{0}^{\prime *} r\right)$.

The bar denotes the complex conjugate.

Property 2.

$$
\left|H_{0}^{(1)}\left(\mathrm{i} \hat{\chi}_{0} r\right)\right|^{2}=H_{0}^{(1)}\left(\mathrm{i} \hat{\chi}_{0} r\right) H_{0}^{(2)}\left(-\mathrm{i} \hat{\chi}_{0}^{*} r\right) .
$$

$H_{0}^{(1)}$ and $H_{0}^{(2)}$ are Hankel functions of zero order and first and second kind, respectively.

By virtue of property 1 and the above calculation $I_{J}$ can be explicitly expressed as

$$
\begin{aligned}
I_{J}= & \left(R / \hat{\chi}_{0}^{\prime 2}-\hat{\chi}_{0}^{\prime * 2}\right)\left[\hat{\chi}_{0}^{\prime} J_{1}\left(\hat{\chi}_{0}^{\prime} R\right) J_{0}\left(\hat{\chi}_{0}^{\prime *} R\right)\right. \\
& \left.-\hat{\chi}_{0}^{\prime *} J_{0}\left(\hat{\chi}_{0}^{\prime} R\right) J_{1}\left(\hat{\chi}_{0}^{\prime *} R\right)\right] .
\end{aligned}
$$

Also, by virtue of property 2 , we can obtain the explicit expression for the integral

$$
I_{H}^{(1)}=\int_{0}^{R} r \mathrm{~d} r\left|H_{0}^{(1)}\right|^{2}
$$

with

$$
\begin{aligned}
I_{H}^{(1)}= & {\left[R /\left(\mathrm{i} \hat{\chi}_{0}\right)^{2}-\left(-\mathrm{i} \hat{\chi}_{0}^{*}\right)^{2}\right] } \\
& \times\left[\mathrm{i} \hat{\chi}_{0} H_{1}^{(1)}\left(\mathrm{i} \hat{\chi}_{0} R\right) H_{0}^{(2)}\left(-\mathrm{i} \hat{\chi}_{0}^{*} R\right)\right. \\
& \left.-(-\mathrm{i}) \hat{\chi}_{0}^{*} H_{0}^{(1)}\left(\mathrm{i} \hat{\chi}_{0} R\right) H_{1}^{(2)}\left(-\mathrm{i} \hat{\chi}_{0}^{*} R\right)\right]+\Lambda_{H}
\end{aligned}
$$

and $\Lambda_{H} \neq 0$, due to the particular behaviour of the function at the limit, so that no cancellations occur (see below).

On the other hand $\int_{0}^{\infty} \mathrm{d} r r\left|H_{0}^{(1)}\right|^{2}$ can be interpreted as an improper integral, as the limit

$$
\int_{0}^{\infty} \mathrm{d} r r\left|H_{0}^{(1)}\right|^{2}=\lim _{R \rightarrow \infty}\left(I_{H}^{(1)}-\Lambda_{H}\right)+\Lambda_{H} .
$$

The validity of equation (36) can be proven as follows. We substitute $H_{0}^{(1)}, H_{1}^{(1)}, H_{0}^{(2)}$ and $H_{1}^{(2)}$ in equation (35) by their asymptotic expansion for large arguments (equations 9.2.3 and 9.2.4 of Abramowitz and Stegun (1972)). For the Hankel functions of the first kind we obtain

$$
\begin{aligned}
& H_{0}^{(1)}\left(\mathrm{i} \hat{\chi}_{0} R\right) \sim\left(2 / \pi \mathrm{i} \hat{\chi}_{0} R\right)^{1 / 2} \\
& \quad \times \exp \left(-\mathrm{i} \hat{\chi}_{0}^{\mathrm{i}} R\right) \exp \left(-\hat{\chi}_{0}^{\mathrm{r}} R\right) \exp (-\pi / 4)
\end{aligned}
$$

and

$$
\begin{aligned}
& H_{1}^{(1)}\left(\mathrm{i} \hat{\chi}_{0} R\right) \sim\left(2 / \pi \mathrm{i} \hat{\chi}_{0} R\right)^{1 / 2} \\
& \quad \times \exp \left(-\mathrm{i} \hat{\chi}_{0}^{\mathrm{i}} R\right) \exp \left(-\hat{\chi}_{0}^{\mathrm{r}} R\right) \exp (-3 \pi / 4)
\end{aligned}
$$


and for the Hankel functions of second kind

$$
\begin{aligned}
& H_{0}^{(2)}\left(-\mathrm{i} \hat{\chi}_{0}^{*} R\right) \sim\left(-2 / \pi \mathrm{i} \hat{\chi}_{0}^{*} R\right)^{1 / 2} \\
& \quad \times \exp \left(\mathrm{i} \hat{\chi}_{0}^{\mathrm{i}} R\right) \exp \left(-\hat{\chi}_{0}^{\mathrm{r}} R\right) \exp (-\pi / 4)
\end{aligned}
$$

and

$$
\begin{aligned}
H_{1}^{(2)}( & \left.-\mathrm{i} \hat{\chi}_{0}^{*} R\right) \sim\left(-2 / \pi \mathrm{i} \hat{\chi}_{0}^{*} R\right)^{1 / 2} \\
& \times \exp \left(-\mathrm{i} \hat{\chi}_{0}^{\mathrm{i}} R\right) \exp \left(-\hat{\chi}_{0}^{\mathrm{r}} R\right) \exp (-3 \pi / 4) .
\end{aligned}
$$

Then, according to equations (34) and (35), equation (36) reads

$$
\int_{0}^{\infty} \mathrm{d} r r\left|H_{0}^{(1)}\right|^{2}=\Lambda_{H}
$$

In fact, let $R=0, H_{0}^{(1)}$ and $H_{0}^{(2)}$ behave as $c \ln (c r)(c$ is a constant). Then

$$
\int_{0}^{R} r \mathrm{~d} r H_{0}^{(1)} H_{0}^{(2)} \sim c \int_{0}^{R} r \mathrm{~d} r[\ln (c r)]^{2} \rightarrow 0 \quad \text { as } R \rightarrow 0
$$

and hence $I_{H}=0, R=0$, since $r$ vanishes more rapidly than $(\ln r)^{2}$.

On the right-hand side of equation (35) we study the limiting behaviour by applying the approximation for Hankel functions of first and second kind with small argument:

$$
\begin{aligned}
& {\left[R /\left(\mathrm{i} \hat{\chi}_{0}\right)^{2}-\left(-\mathrm{i} \hat{\chi}_{0}^{*}\right)^{2}\right]\left\{\mathrm{i} \hat{\chi}_{0} H_{1}^{(1)} H_{0}^{(2)}-(-\mathrm{i}) \hat{\chi}_{0}^{*} H_{0}^{(1)} H_{1}^{(2)}\right\}} \\
& \quad \rightarrow\left[-1 /\left(\mathrm{i} \hat{\chi}_{0}\right)^{2}-\left(-\mathrm{i} \hat{\chi}_{0}^{*}\right)^{2}\right] \Gamma(1)(2 / \pi)^{2} \\
& \quad \times \ln \left[-\left(\hat{\chi}_{0}^{*} / \hat{\chi}_{0}\right)\right]
\end{aligned}
$$

Here $\Gamma(1)$ represents the gamma function of unit argument $(\Gamma(1)=1)$.

To obtain on the right-hand side of equation (35), an expression consistent with the behaviour of $I_{H}$ at $R=0$, the constant of integration $\Lambda_{H}$ is such that it establishes the cancellation of the constant term in equation (42), then

$$
\Lambda_{H}=\left[-1 /\left(\chi_{0}^{2}-\hat{\chi}_{0}^{* 2}\right)\right] \Gamma(1)(2 / \pi)^{2} \ln \left[-\left(\hat{\chi}_{0}^{*} / \hat{\chi}_{0}\right)\right]
$$

By substituting into equation (41), one obtains the explicit expression for $I_{H}$ (see equation (28)). Numerically, $I_{H}$ can be easily calculated since $\Lambda_{H}$ and $I_{H}^{(1)}$ are known.

\section{Polynomial expansion and computational method}

To evaluate equations (25) and (26) numerically, use of the polynomial expansion for $J_{0}$ and $J_{1}$ has been made (section 9.4 of Abramowitz and Stegun (1972)), with the specification that the variable is now a complex one. We notice that use of standard tables of Bessel functions $J_{0}(z)$ and $J_{1}(z)$ for complex argument (Watson 1942 , National Bureau of Standards 1943), cannot be used here due to the particular values of the argument of the complex variable appearing for a standard absorbing optical waveguide, as in general $\arg \hat{\chi}_{0}^{\prime} R$ cannot be expressed in terms of an entire number of radians (Lakshminarayanan and Calvo 1987). In this special case the condition for the applied polynomial expansion holds as:

$$
-3<|\hat{x}|<+3
$$

with

$$
|\hat{x}|=\left|\hat{\chi}_{0}^{\prime}\right| R=\left[\left(\hat{\chi}_{0}^{\prime r}\right)^{2}+\left(\hat{\chi}_{0}^{\prime i}\right)^{2}\right]^{1 / 2} R
$$

We recall that $\hat{\chi}_{0}^{\prime r}$ gives information on the transmission properties of the waveguide while $\hat{\chi}_{0}^{\prime i}$ is related to the absorption ones. For standard optical waveguides with weak absorption, such as optical photoreceptors, the extinction coefficient is about $K \sim 10^{-3}$ in the visible spectrum (Harosi 1981). In this case, we usually deal with values for $|\hat{x}| \sim 3 R$ ( $R$ being the radius of the core). If the waveguide propagates light under a monomode regime, or quasi-monomode (supporting a low number of bound modes), then $R \sim 1.0 \mu \mathrm{m}$ and we can keep up to a second order of approximation in the polynomial expansion. The numerical equations read

$$
\begin{array}{ll}
J_{0}^{\mathrm{r}}(\hat{x})=1-0.25\left[\left(\hat{\chi}_{0}^{\prime r}\right)^{2}-\left(\hat{\chi}_{0}^{\prime \mathrm{i}}\right)^{2}\right] R^{2} & \\
J_{0}^{\mathrm{i}}(\hat{x})=-0.5 \chi_{0}^{\prime r} \hat{\chi}_{0}^{\prime \mathrm{i}} \cdot R^{2} \quad & \left|\hat{\chi}_{0}^{\prime}\right| R \leqslant 3 \\
& \left|\hat{\chi}_{0}^{\prime}\right| R \leqslant 3 .
\end{array}
$$

And in a similar way we obtain for $J_{1}$ :

$$
\begin{array}{r}
J_{1}^{\mathrm{r}}(x)=\hat{\chi}_{0}^{\prime r} R\left\{0.5-0.0625 R^{2}\left[\left(\hat{\chi}_{0}^{\prime \mathrm{r}}\right)^{2}-3\left(\hat{\chi}_{0}^{\prime \mathrm{i}}\right)^{2}\right]\right\} \\
\left|\hat{\chi}_{0}^{\prime}\right| R \geqslant 3 \\
J_{1}^{\mathrm{i}}(x)=\hat{\chi}_{0}^{\prime \mathrm{i}} R\left\{0.5-0.0625 R^{2}\left[3\left(\hat{\chi}_{0}^{\prime \mathrm{r}}\right)^{2}-\left(\hat{\chi}_{0}^{\prime}\right)^{2}\right]\right\} \\
\left|\hat{\chi}_{0}^{\prime}\right| R \leqslant 3 .
\end{array}
$$

The consequences of using this approximation will be discussed later.

Additional polynomial expansions for $3<|\hat{x}|<+\infty$ can be used for both $J_{0}$ and $J_{1}$ by keeping the same order of approximation as well. In equations (44)-(47), the superscripts ' $r$ ' and ' $i$ ' denote the real and imaginary parts for $J_{0}$ and $J_{1}$ respectively.

The numerical estimates are displayed in figure 2 . In this figure, the radial distribution for $J_{0}^{\mathrm{r}}, J_{0}^{\mathrm{i}}, J_{1}^{\mathrm{r}}$ and $J_{1}^{\mathrm{i}}$ are displayed, together with the cross-product $J_{0}^{\mathrm{r}} J_{1}^{\mathrm{r}}+J_{0}^{\mathrm{i}} J_{1}^{\mathrm{i}}$, which contributes to the total fraction of the confined power $P_{0}^{\mathrm{F}}$ through equation (33). We observe the correct behaviour for $J_{0}^{\mathrm{r}}$ and $J_{1}^{\mathrm{r}}$ thus validating the polynomial expansion previously established. Also, we notice the small contribution to $P_{0}^{F}$ coming from $J_{0}^{\mathrm{i}}$ and $J_{1}^{\mathrm{i}}$, due to the weak absorption condition that has been assumed.

In figure 3 we show the distribution of the complex Bessel functions versus the dimensionless parameter $a=\hat{\chi}_{0}{ }^{r} R$. This is in fact the real parameter to be used in the comparison of the distribution of the density of energy ( $P_{0}^{\mathrm{F}} /$ unit area), between dielectric (absorptionless) and absorbing waveguides. 


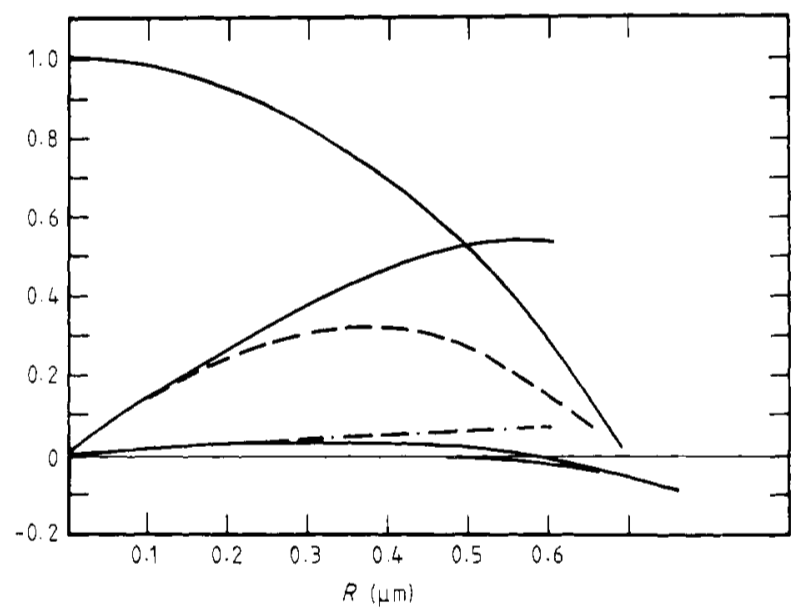

Figure 2. Radial distribution of the real and imaginary parts of the complex functions $J_{0}$ and $J_{1}$ (full curves). The crossproducts $J_{0}^{r} J_{1}^{r}+J_{0}^{i} J_{1}^{i}$ (broken curve) and $J_{0}^{r} J_{1}^{i}-J_{0}^{i} J_{1}^{r}$ (chain curve) are shown since both of these terms contribute to the fraction of the confined power $P_{0}^{F}$ within the absorbing waveguide. The modal parameter $\hat{\chi}_{0}^{\prime}$ is fixed as $\hat{\chi}_{0}^{\prime r}=2.8$ and $\hat{\chi}_{0}^{\prime \prime}=3 \times 10^{-2}$, then $\left|\hat{\chi}_{0}^{\prime}\right| R<3.0$.

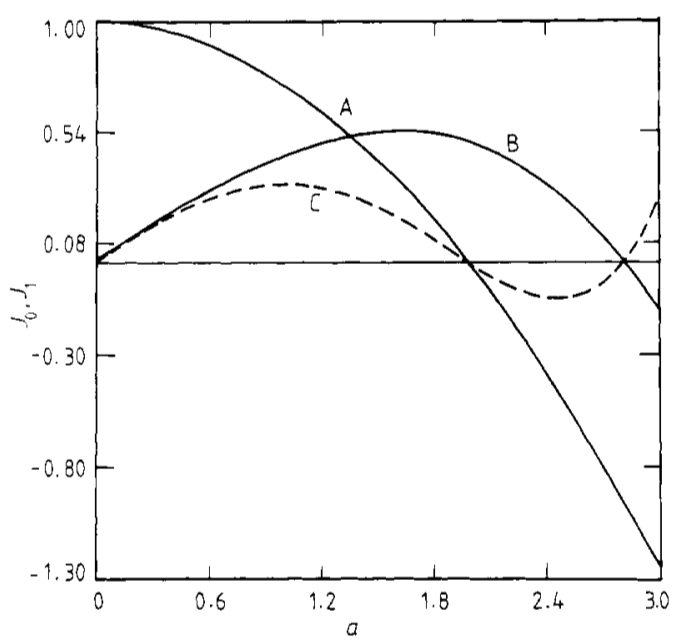

Figure 3. Numerical behaviour of the complex functions $J_{0}$ and $J_{1}$ versus the dimensionless parameter $a=\hat{\chi}_{0}^{\prime r} R$. Note the small contribution of $J_{0}^{i}\left(J_{1}^{j}\right.$ values are not displayed since they are extremely small). For values of $a>3.0$, the functions $J_{0}^{r}$ and $J_{1}^{r}$ coincide. Curves $A$ and $B$ show $J_{0}$ and $J_{1}$, curve $C$ shows $J_{0}^{r} J_{1}+J_{0}^{i} J_{1}^{i}$.

\section{Summary and conclusions}

We have defined the exact analytical representation for the initial field striking an absorbing waveguide. The uniqueness of the decomposition is also discussed. The mathematical treatment leads to the exact formulation for the fraction of the confined power, and, because of the absorption physical conditions, Bessel and Hankel functions with complex arguments are involved. The integration of these types of functions is not trivial, and details on some applicable methods are presented. For completeness, some comments on properties of Bessel and Hankel functions with complex argument are included.

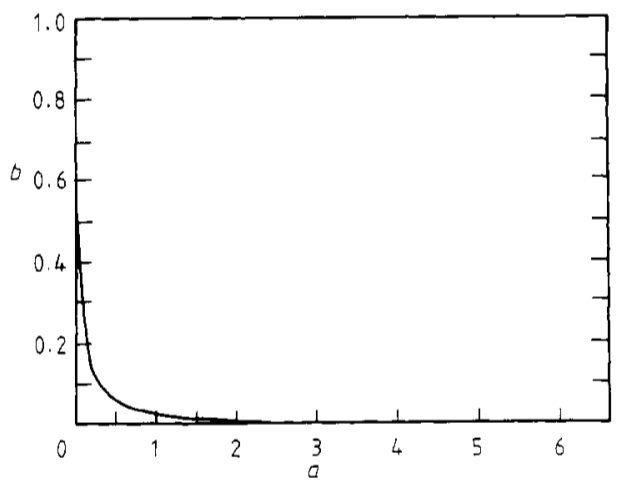

Figure 4. Variation of the dimensioniess parameter $b=\hat{\chi}_{0}^{\prime} R$ against $a=\hat{\chi}_{0}^{\prime} R$, showing the rapid decrease of the function. For $a>2.0$ the parameter $b$ is negligible.

Also, a discussion on some useful approximations for computation of the real and imaginary parts of $J_{0}$ and $J_{1}$ through polynomial expansions is given, together with some numerical calculations showing the correctness in the behaviour of both $J_{0}^{\mathrm{r}}$ and $J_{1}^{\mathrm{r}}$, as well as the small contribution coming from $J_{0}^{\mathrm{i}}$ and $J_{1}^{\mathrm{i}}$ due to the previously established weak absorption conditions. These results lead to a complete interpretation from an analytical solution of the fraction of the confined power and density of energy in a weak absorbing optical waveguide.

\section{Acknowledgments}

MLC wants to thank the US-Spanish Joint Committee for Scientific and Technological Cooperation for financial support. This work was supported in part by a National Eye Institute grant EY03674 from the National Institutes of Health, Bethesda, MD. We also acknowledge the interest and support of J M Enoch. Thanks also to Christina Vigil for working on the manuscript.

\section{References}

Abramowitz M and Stegun I (ed.) 1972 Handbook of Mathematical Functions (New York: Dover) 9th edn Alvarez-Estrada R F and Calvo M L $1984 J$. Phys. D: Appl. Phys. 17475

Alvarez-Estrada R F, Calvo M L and Juncos P 1980 Opt. Acta 271367

Bateman Manuscript, Tables of Integral Transforms 1954 (New York: McGraw-Hill) vol 2 p 90

Calvo M L and Duran A 1975 Nuovo Cimento B 29277

Calvo M L and Lakshminarayanan V 1987 J. Opt. Soc. Am. A 41037

Crow J D. Borrelli N F, Seward T P III and Chodak J 1975 Appl. Opt 14580

Enoch J M and Tobey F L Jr (ed.) 1981 Vertebrate Photoreceptor Optics (Berlin: Springer)

Harosi F I 1981 Microspectrophotometry and optical phenomena: Birefringence, dichroism and anomalous dispersion Vertebrate Photoreceptor Optics ed. J M Enoch and F L Tobey Jr (Berlin: Springer) ch. 9 
Lakshminarayanan V and Calvo M L 1987 J. Opt. Soc. Am. A 42133

Marcuse D 1972 Light Transmission Optics (New York: Van Nostrand)

Mattern P L, Watkins L M, Skoog C D, Brandon J R and Bargis E H 1974 IEEE Trans. Nucl. Sci. NS-21 81

Morse P M and Feshbach H 1953 Methods of Theoretical Physics (New York: McGraw-Hill) vol 1

National Bureau of Standards 1943 Table of the Bessel
Functions $J_{0}(z)$ and $J_{1}(z)$ for Complex Argument (New York: NBS, Columbia University Press)

Pask C and Barrell K F 1981 Biol. Cyb. 361

Snyder A W 1969 IEEE Trans. Microwave Theory and Tech. MTT-17 1138

Snyder A W and Love J D 1983 Optical Waveguide Theory (London: Chapman and Hall)

Watson G N 1942 A Treatise on the Theory of Bessel Functions (London: Cambridge University Press) 2nd edn 